\title{
Mixing augmentation mechanism induced by the pseudo shock wave in transverse gaseous injection flow fields
}

\author{
Wei Huang ${ }^{1 *}$, Ming-hui $\mathrm{Li}^{2}, \mathrm{Li}_{\text {Yan }}{ }^{1}$ \\ 1. Science and Technology on Scramjet Laboratory, National University of Defense Technology, Changsha, \\ Hunan 410073, People's Republic of China
}

2. China Aerodynamics R\&D Center, Mianyang, Sichuan 621000, People's Republic of China

\begin{abstract}
The mixing process between the injectant and air is very crucial for the engineering implementation of the scramjet engine, and this is due to the very short resident time of fuel in supersonic flows. In the current
\end{abstract} study, the three-dimensional Reynolds-average Navier-Stokes (RANS) equations coupled with the two equation $k$ - $\omega$ shear stress transport (SST) turbulence model have been employed to investigate the transverse injection flow field with the pseudo shock wave induced by the high back pressure, and the freestream Mach number is 3.75. At the same time, the influence of the back pressure on the flow field properties has been evaluated as well. The obtained results show that the pseudo shock wave induced by the back pressure plays an important role in the mixing enhancement between the injectant and air. When the back pressure ratio is larger than 5.0, the mixing efficiency increases with the increase of the back pressure ratio. However, when the back pressure ratio is 3.0, the near-field mixing process has been improved, and accordingly its mixing efficiency in this region is larger than the benchmark. This implies that the intense combustion downstream of the injector can enhance the mixing process between the injectant and air, and the mixing and combustion process can be enhanced mutually. When the pseudo shock wave has been pushed upstream of the wall orifice, more injectant has been brought into the separation zone upstream of the injector, and this is beneficial for the mixing process between the

* Associate Professor, Corresponding author, E-mail: gladrain2001@163.com, Phone: +86 731 84576447, Fax: +86 73184512301 
injectant and air.

Keywords: Supersonic mixing; transverse injection; pseudo shock wave; back pressure; mixing enhancement; scramjet engine

\section{Introduction}

Scramjet fuel injection has received considerable attention since the mid-1950s [1]. Glagolev and his coworkers [2-3] investigated supersonic flow with freestream Mach number being 3.0 past an air jet issuing at the speed of sound, and the obtained quantitative data are useful for further study of the same phenomenon. Recently, numerous fuel injector concepts have been proposed and investigated, especially for the cantilevered ramp injector located in the forebody/inlet of hypersonic vehicles [4-5] and pylon used as the vortex generator ahead of the orifice [6-8]. However, these devices are intrusive to the flow, generate drag, and require cooling [9]. Therefore, the flush-wall injection continues to remain one of the simplest and most promising strategies to enhance the mixing process between the air and injectant in supersonic flows, and it attracts an increasing attention since the early sixties [10-17]. Huang and Yan [18] provided a detailed review on the transverse injection flow field in supersonic flows, and its four aspects were summarized, namely the jet-to-crossflow pressure ratio, the geometric configuration of the injection port, the number of injection ports and the injection angle. A stinger-shaped injector with a sharp leading edge in front of a streamwise slit was employed by Kouchi et al. [19] to enhance the mixing and combustion process between the fuel and air, and the measured data were compared with those of a conventional circular injector.

Further, the oblique shock wave is proved to be an important issue on the mixing enhancement, and this is due to the strong axial vortices generated by the interaction between the mixing layer and the oblique shock wave. The axial vortices stretch the fuel/air interface [20]. Schetz et al. [21], Huang et al. [22-23] and 
Gerdroodbary et al. [24] investigated the shock wave effect on the supersonic mixing in scramjet engines experimentally and numerically, and they found that the impinging shock wave reduces the penetration and increases the mixing for the injectants irrespective of the molecular weights. Zare-Behtash et al. [25] studied the effect of an impinging shock wave on the jet-cavity interaction experimentally, and a relatively uniform velocity distribution within the cavity was induced by the interaction between the shock wave and the transverse jet. Based on his recent research works, Huang [26] gave a comprehensive survey on transverse jet in supersonic crossflows, and the research progress on two main additional topics has been included, namely the interaction between jet and vortex generator, and the interaction between jet and shock wave.

At the same time, when the combustion process occurs downstream of the fuel injection location, the supersonic mixing process would be affected as well, and this may be due to the high back pressure generated by the intensive combustion between the fuel and air. Then, the pseudo shock wave which is common in the isolator [27] would be formed if the back pressure is high enough, and it has a large impact on the deflagration-to-detonation transition in metastable systems as well [28]. The pseudo shock wave could lower the flow velocity of the mainstream and increase the size of the counter-rotating vortex pair [29]. In order to clarify the mixing enhancement mechanism induced by the pseudo shock wave, more detailed information is required about the flowfield generated by the pseudo shock wave. Unfortunately, the experimental investigations of these complex flows are costly to perform, and the computational studies can often offer insight into the mechanics of these flows [30].

Ogawa et al. [31] conducted cold flow experiments to investigate the mixing process of fuel in the duct flow with a pseudo shock wave presents. Kouchi et al. [32] took high-speed schlieren movies of transverse injection flow fields with and without the pseudo shock wave, and they observed that the injectant plume penetrates much deeper into the mainstream when a pseudo shock wave is present. 
In the current study, the transverse injection flow field with the pseudo shock wave has been investigated numerically, and the influence of the pseudo shock wave on the supersonic mixing between the fuel and air has been evaluated. The pseudo shock wave is formed by setting a high back pressure at the exit of the channel, and the back pressure ratio is set to be 3.0, 5.0, 8.0, 10.0 and 15.0.

\section{Physical model and numerical approach}

\subsection{Physical model}

The test section is a straight channel with a square cross section of $30 \times 30 \mathrm{~mm}$, and its length is $290 \mathrm{~mm}$. Hydrogen is injected perpendicular to the test section from a sonic circular orifice $1.0 \mathrm{~mm}$ in diameter, positioned on the bottom wall of the section, see Fig.1. Fig.1 shows the schematic diagram of the physical model studied in this paper. The injection port is located on the centerline of the wall, $200 \mathrm{~mm}$ downstream of the entrance of the channel.

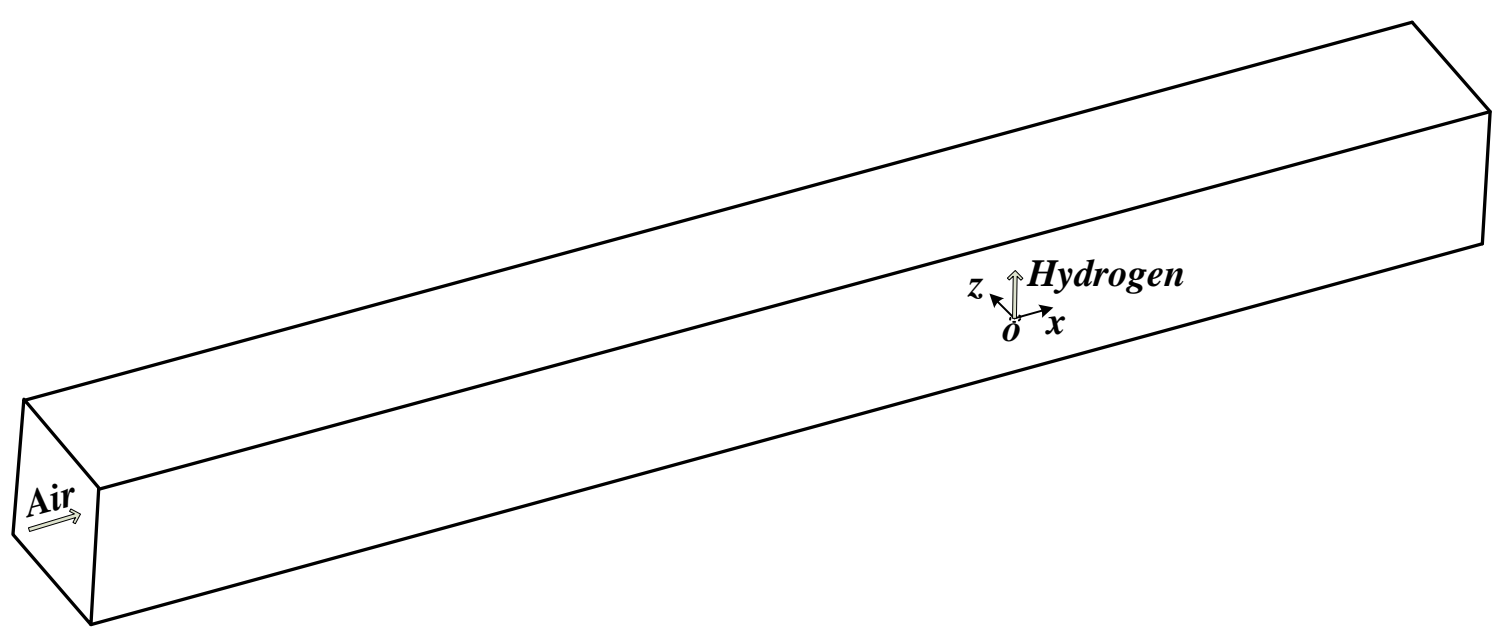

Fig.1 Schematic diagram of the transverse injection flow field with the pseudo shock wave.

The air properties are set to be a Mach number $M_{\infty}$ of 3.75, a static pressure $P_{\infty}$ of $11090 \mathrm{~Pa}$ and a static temperature $T_{\infty}$ of $78.43 \mathrm{~K}$. The air flows from left to right. The jet flow Mach number $M_{\mathrm{j}}$ is set to be 1.0 , with a static temperature $T_{\mathrm{j}}=249 \mathrm{~K}$ and a jet-to-crossflow pressure ratio $P_{\mathrm{j}} / P_{\infty}=10.29$. These parameters are the same 
as those set by Aso et al. [33]. A Cartesian coordinate system, with its origin at the center point of the jet orifice, see Fig.1, is employed to represent the predicted results, where the streamwise direction is along the $x$ axis, the direction of injection is along the $y$ axis, and the spanwise direction is along the $z$ axis. In order to evaluate the effect of back pressure on the supersonic mixing process between the air and fuel, a high back pressure is provided at the exit of the channel, and the back pressure ratio $\left(P_{\mathrm{b}} / P_{\infty}\right)$ is set to be 3.0, 5.0, 8.0, 10.0 and 15.0.

\subsection{Numerical approach}

The steady state computational data have been generated using FLUENT version 6.3.26, and the mesh generation has used the commercial software Gambit [34]. A Dell workstation at Science and Technology on Scramjet Laboratory, using up to 32 processors, has provided a parallel computing environment for flow solutions.

For this study, the three-dimensional Reynolds-average Navier-Stokes (RANS) equations have been solved using a coupled, implicit, second-order upwind solver, and it is a steady-state model. Cell fluxes have been computed using an AUSM scheme, and the viscosity has been determined using mass-weighted-mixing-law. The turbulent viscosity is computed by two equation $k-\omega$ shear stress transport (SST) turbulence model, and the transport equations for $k$ and $\omega$ can refer to Ref.[35]. The SST model was utilized successfully in previous studies on transverse injection flow [36-37]. The Courant-Friedrichs-Levy (CFL) number remains at 0.5 with suitable under-relaxation factors to ensure stability. The no-slip conditions $(u=v=w=0.0)$ are assumed for wall boundaries.

The grid density is clustered towards the injection port for all grids and relaxed towards outlet, see Fig.2, and Fig.2 depicts the schematic diagram of the whole grid system employed in the current study. A boundary layer grid is generated on the walls with a first cell height of $0.001 \mathrm{~mm}$, which results in a suitable value of $y^{+}$for all 
of the flow fields, and its maximum value is less than 2.0. Because of its configuration being symmetric, only half of the model is simulated numerically, and a nominal three-dimensional structured grid with 0.83 million cells is selected. $y^{+}$is a non-dimensional parameter defined by:

$$
y^{+}=\frac{\rho u_{\tau} y_{P}}{\mu}
$$

Herein, $u_{\tau}$ is the friction velocity, $y_{P}$ is the distance from point $P$ to the wall, $\rho$ is the fluid density, and $\mu$ is the fluid viscosity at point $P$.

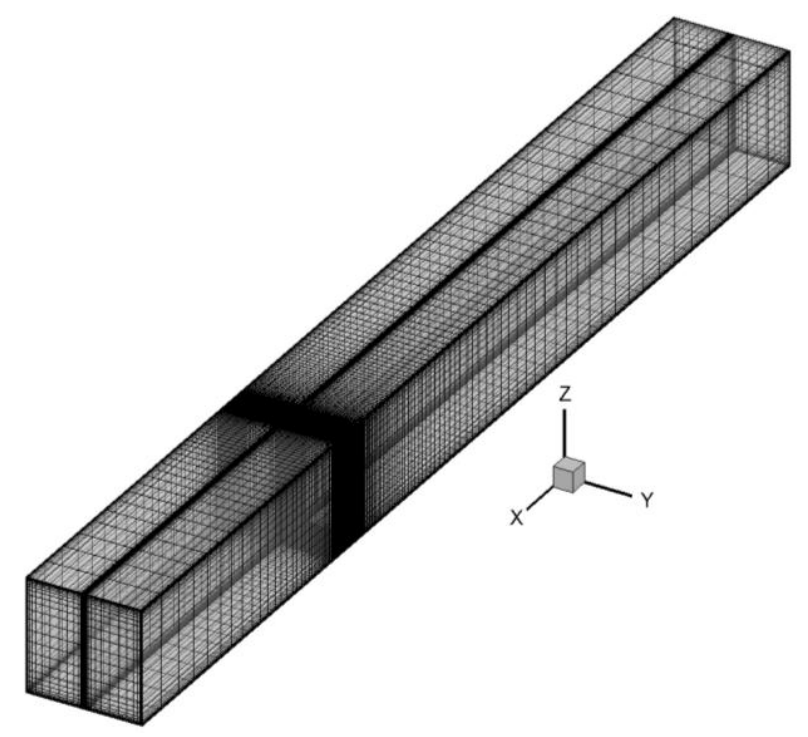

Fig.2 Schematic diagram of the whole grid system employed in the current study.

\section{Code validation and grid independency analysis}

In this section, three grid scales have been used to conduct the grid independency analysis for the model without the back pressures, namely the coarse, moderate and refined grids, and their numbers of cells are nearly 0.25 million, 0.83 million and 2.12 million for half of the model, respectively. Fig. 3 represents the wall static pressure distributions along the symmetric line without the back pressures, and it is observed that the predicted results obtained by the moderate grid show better agreement than those obtained by the refined grid. This is related to the parallel computing environment and the numerical approach used [38]. The accumulation of 
stochastic error is proportional to the number of time steps and depends on the accuracy of the scheme and the approximation error [39]. The separation length is over-predicted by the coarse grid. However, the area of its recirculation zone downstream of the wall orifice is under-predicted. This may imply that the moderate grid with 0.83 million cells is suitable for the simulation.

Further, the validation metric is used to quantify the level of agreement between the computational results, and it is applied to the internal compression flow of a typical supersonic inlet successfully [40]. Its definition is as follows [41]:

$$
V=1-\frac{1}{N} \sum_{i=1}^{N} \operatorname{Tanh}\left|\frac{y\left(x_{i}\right)-Y\left(x_{i}\right)}{Y\left(x_{i}\right)}\right|
$$

Here, $V$ is the validation metric, and $Y\left(x_{i}\right)$ is the benchmark corresponding to the dependent variable $y$ at $x=$ $x_{i}$ for $i=1,2,3 \ldots N$. This metric has many advantages, and more information can refer to Ref. [41]. In this article, the predicted results obtained by the moderate grid have been chosen as the benchmark for lacking of the available experimental data. The validation metrics for the predicted results obtained by the refined and coarse grids are 0.951 and 0.922 , respectively, and this implies that the predicted results obtained by the refined grid show better agreement with those obtained by the coarse grid. This conclusion is consistent with that observed in Fig.3, and the moderate grid is proved to be suitable for this model employed in the current study.

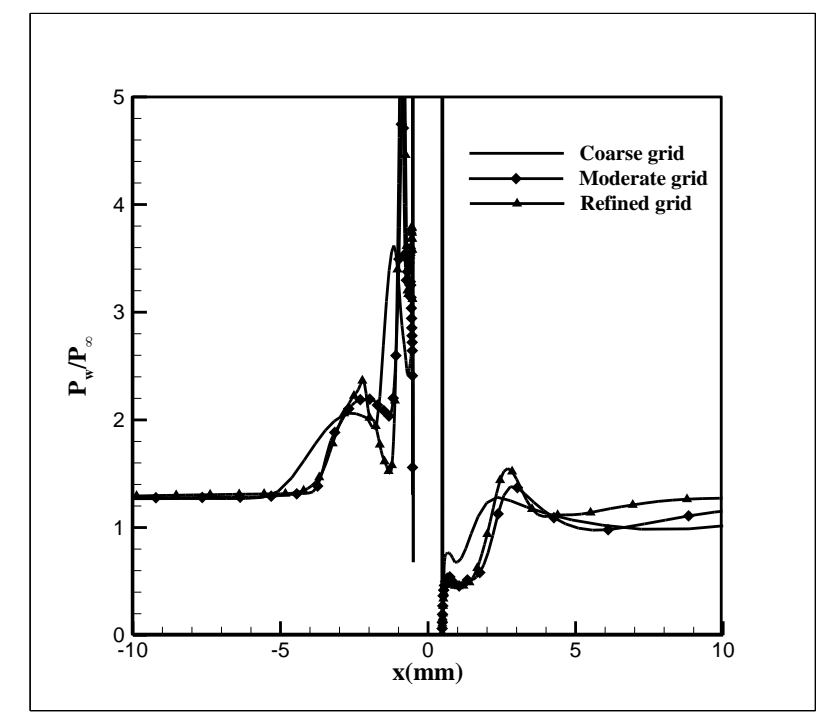

Fig.3 Wall static pressure distributions along the symmetric line without the back pressure. 
At the same time, the experimental model, as studies by Aso et al. [33] is utilized to provide data for validation of the numerical method as well, and the injection port is a slot not an orifice. Fig. 4 shows the wall static pressure comparisons with the jet-to-crossflow pressure ratio being 10.29 in the three-dimensional transverse injection flow field without the pseudo shock wave, and it is obvious that the predicted results show good agreement with the experimental data obtained by Aso et al. [33]. The grid scale has only a slight impact on the wall static pressure distribution for this model being nearly two-dimensional. More information can refer to Ref.[36].

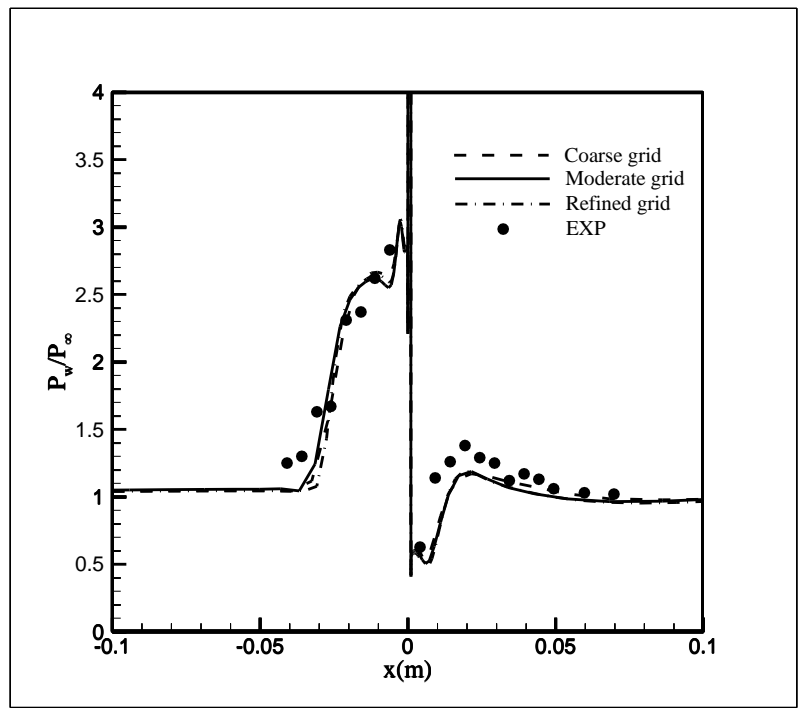

Fig.4 Wall static pressure comparisons with the jet-to-crossflow pressure ratio being 10.29 in three-dimensional transverse injection flow field without the pseudo shock wave [36].

\section{Results and discussion}

Fig.5 depicts the comparison of wall static pressure distributions along the symmetric line with different back pressure ratios, and the case without the back pressure has been chosen as the benchmark. It is clearly observed that the wall static pressure increases with the increase of the back pressure when the back pressure ratio is more than 3.0. The wall static pressure distribution of the case with the back pressure ratio being 3.0 is nearly the same as that of the case without the back pressure, and this implies that the overall properties of the flow field in 
the flowpath have not been affect by the back pressure when its value is not large enough, namely $P_{\mathrm{b}} / P_{\infty}=3.0$ in the range considered in the current study. This conclusion can also be deducted from the Mach number and hydrogen mole fraction contours, see Figs.6 (a) and 7 (a). It is obvious that the flow field of the case with the back pressure ratio being 3.0 is nearly the same as that of the case without the back pressure, and the flow field structure generated by the transverse gaseous injection has not been broken down by the back pressure. Figs. 6 and 7 represent the Mach number contour and hydrogen mole fraction contour comparisons at the symmetric plane with different back pressure ratios, respectively.

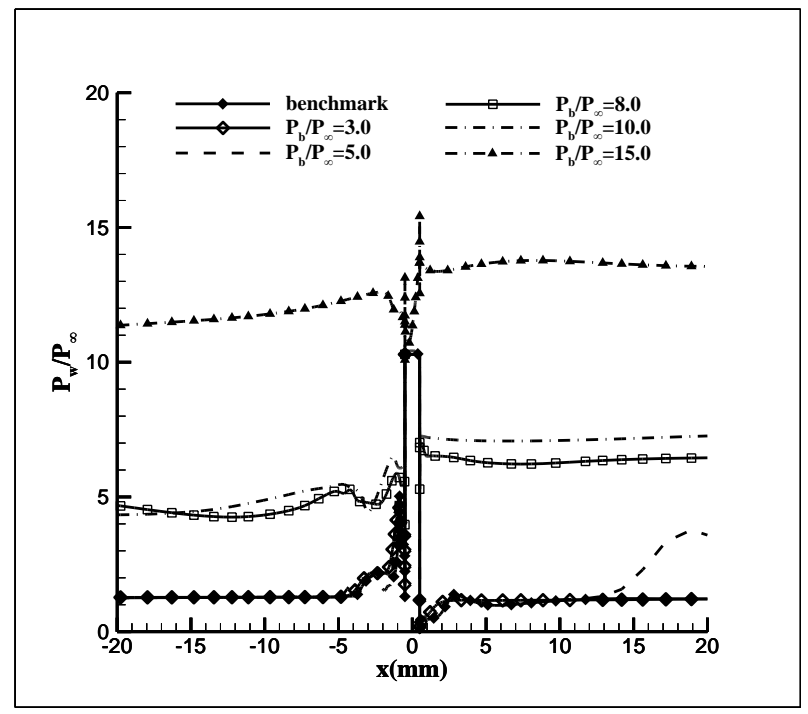

Fig.5 Comparison of wall static pressure distributions along the symmetric line with different back pressure
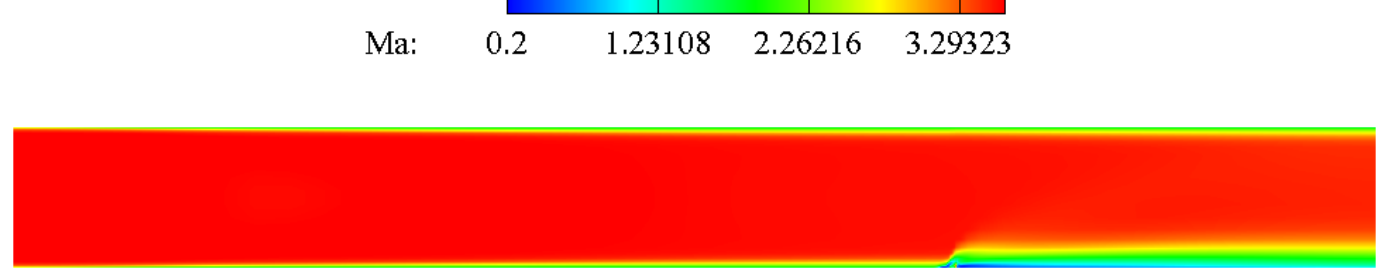

(a) $P_{\mathrm{b}} / P_{\infty}=3.0$

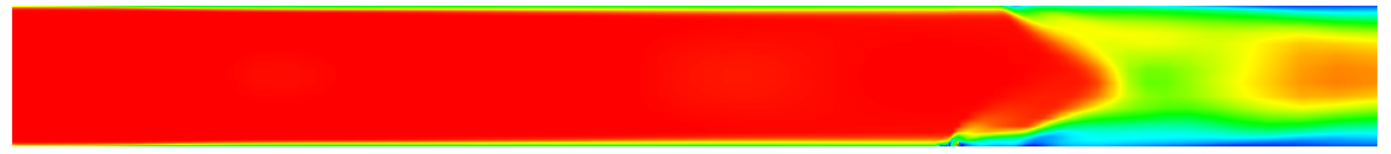

(b) $P_{\mathrm{b}} / P_{\infty}=5.0$ 
(c) $P_{\mathrm{b}} / P_{\infty}=8.0$

(d) $P_{\mathrm{b}} / P_{\infty}=10.0$

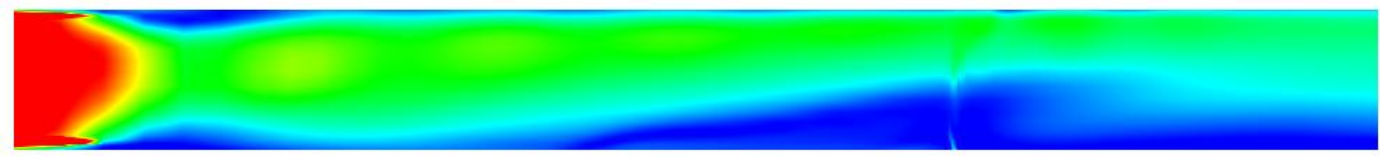

(e) $P_{\mathrm{b}} / P_{\infty}=15.0$

Fig.6 Mach number contour comparison at the symmetric plane with different back pressure ratios, (a) $P_{\mathrm{b}} / P_{\infty}=$ 3.0, (b) $P_{\mathrm{b}} / P_{\infty}=5.0$, (c) $P_{\mathrm{b}} / P_{\infty}=8.0$, (d) $P_{\mathrm{b}} / P_{\infty}=10.0$ and (e) $P_{\mathrm{b}} / P_{\infty}=15.0$.
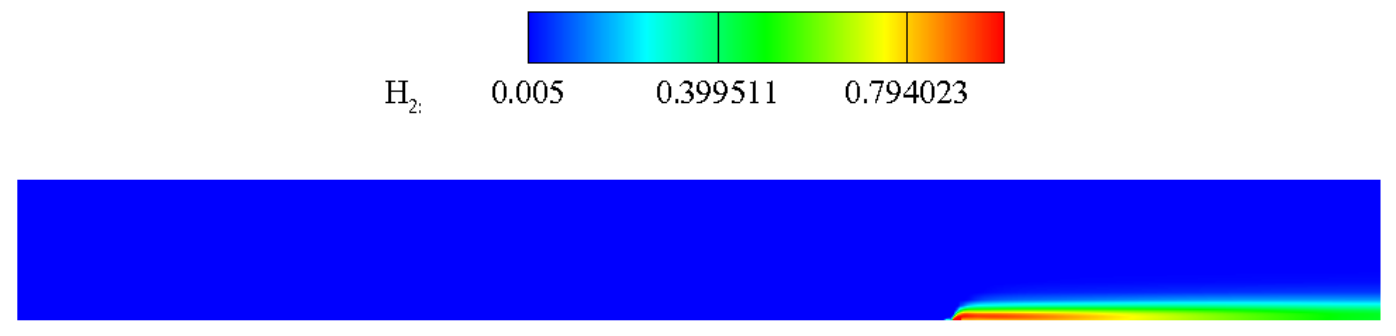

(a) $P_{\mathrm{b}} / P_{\infty}=3.0$

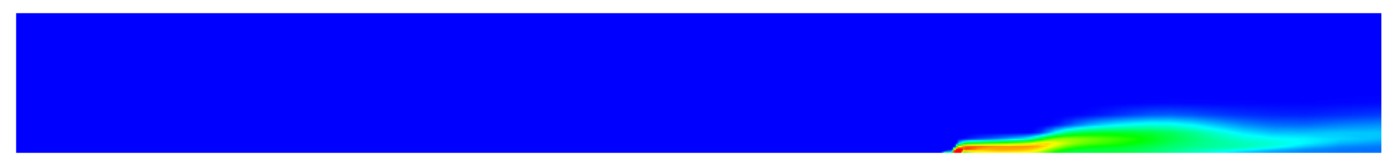

(b) $P_{\mathrm{b}} / P_{\infty}=5.0$

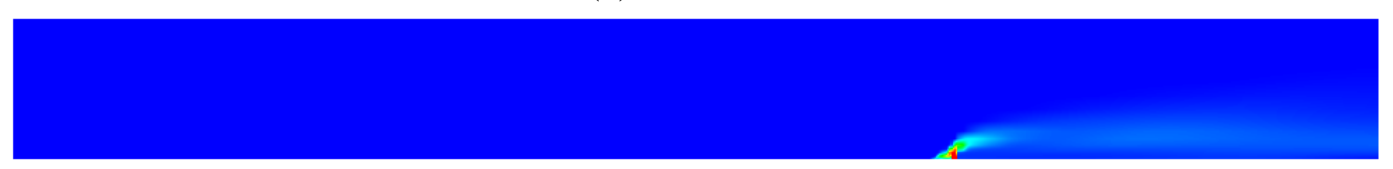

(c) $P_{\mathrm{b}} / P_{\infty}=8.0$

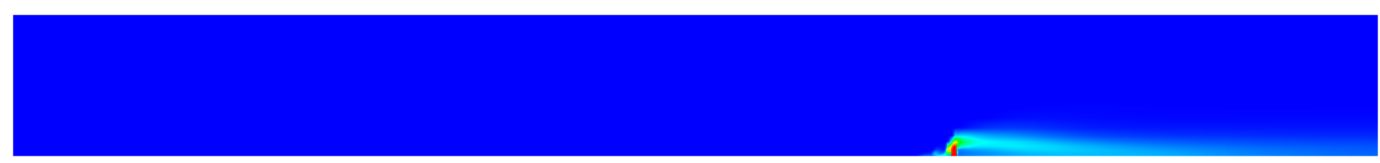

(d) $P_{\mathrm{b}} / P_{\infty}=10.0$

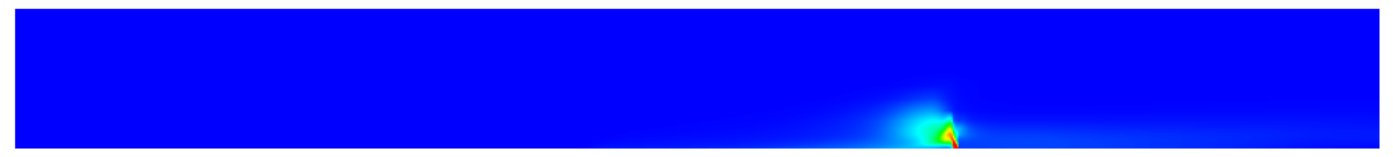

(e) $P_{\mathrm{b}} / P_{\infty}=15.0$

Fig.7 Hydrogen mole fraction contour comparison at the symmetric plane with different back pressure ratios, (a) 


$$
P_{\mathrm{b}} / P_{\infty}=3.0, \text { (b) } P_{\mathrm{b}} / P_{\infty}=5.0 \text {, (c) } P_{\mathrm{b}} / P_{\infty}=8.0, \text { (d) } P_{\mathrm{b}} / P_{\infty}=10.0 \text { and (e) } P_{\mathrm{b}} / P_{\infty}=15.0 \text {. }
$$

When the back pressure ratio is 5.0 , the static pressure in the vicinity of the exit of the channel has been affected first, see Fig.5, and its value increases sharply at $x=15 \mathrm{~mm}$. Accordingly, a shock wave train is formed in the flowpath, see Fig.6 (b), and the hydrogen mole fraction distribution is affected by the shock wave train as well, see Fig.7 (b). When the back pressure ratio is larger than 5.0, the static pressures upstream and downstream of the injector all increase due to the high back pressure, and the shock wave train has been pushed upstream of the injector, see Figs.6(c), (d) and (e). The separation and recirculation zones upstream and downstream of the wall orifice have been broken down, and they become larger accordingly. Then, some injectant has been brought upstream of the injector, see Figs.7(c), (d) and (e), and the hydrogen mole fraction decreases sharply due to the effect of the shock wave train.

The hydrogen mole fraction contours at $x=20 \mathrm{~mm}$ with different back pressure ratios have been laid out in order to illustrate the effect of the back pressure on the flow field properties of the transverse gaseous injection flow field further, see Fig.8. Fig. 8 shows the hydrogen mole fraction contour comparison at $x=20 \mathrm{~mm}$ with different back pressure ratios, and it is clearly observed that the hydrogen mole fraction decreases sharply with the increase of the back pressure. When the back pressure ratio is 15.0 , the hydrogen nearly disappears, and this may imply that the injectant and air almost mix completely. At the same time, we observe that the fuel plume area deceases as well, and the peach-shaped plume has been broken down by the shock wave train. 


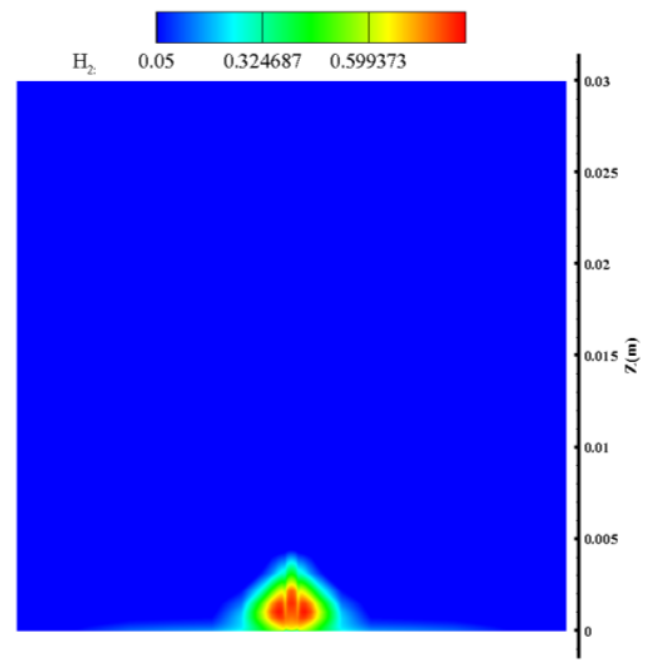

(a) Benchmark

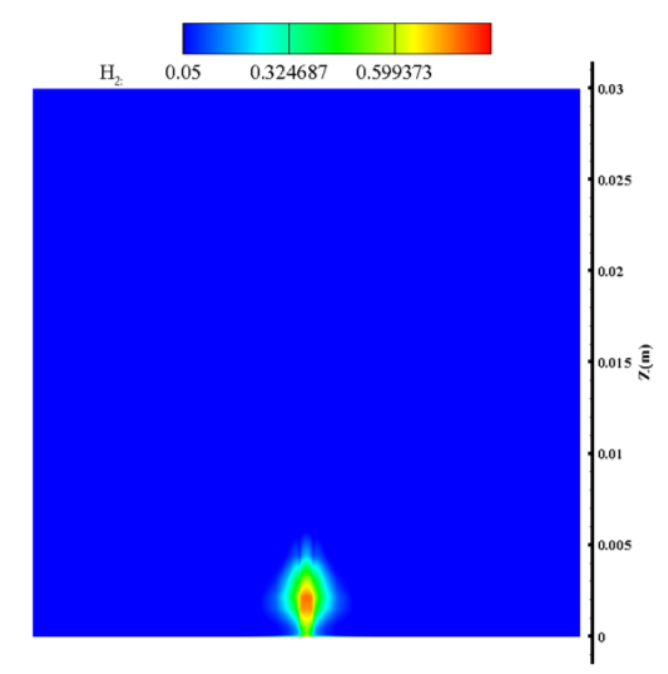

(c) $P_{\mathrm{b}} / P_{\infty}=5.0$

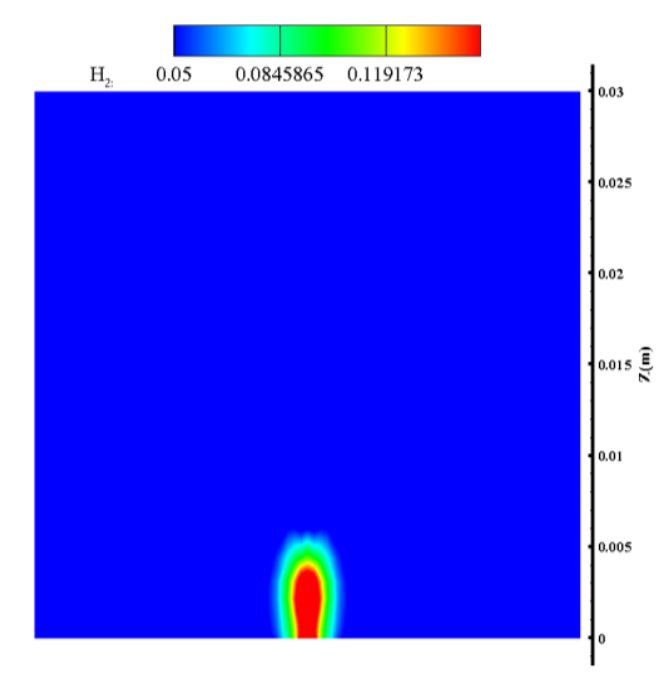

(e) $P_{\mathrm{b}} / P_{\infty}=10.0$

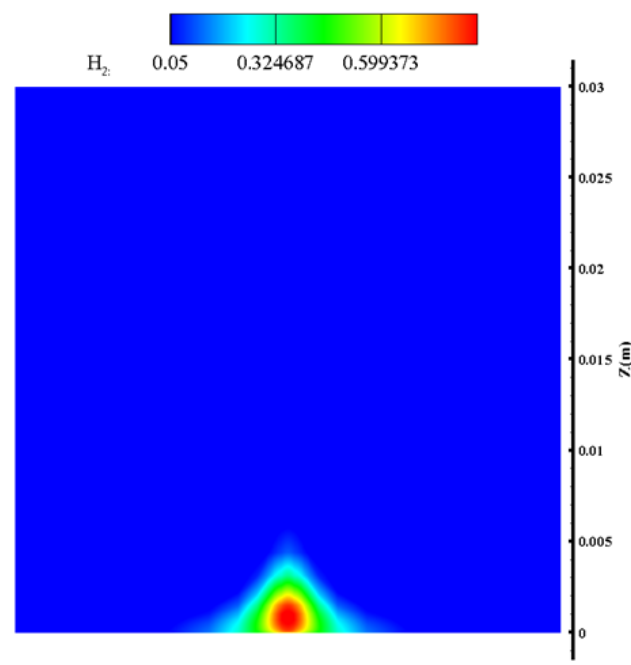

(b) $P_{\mathrm{b}} / P_{\infty}=3.0$

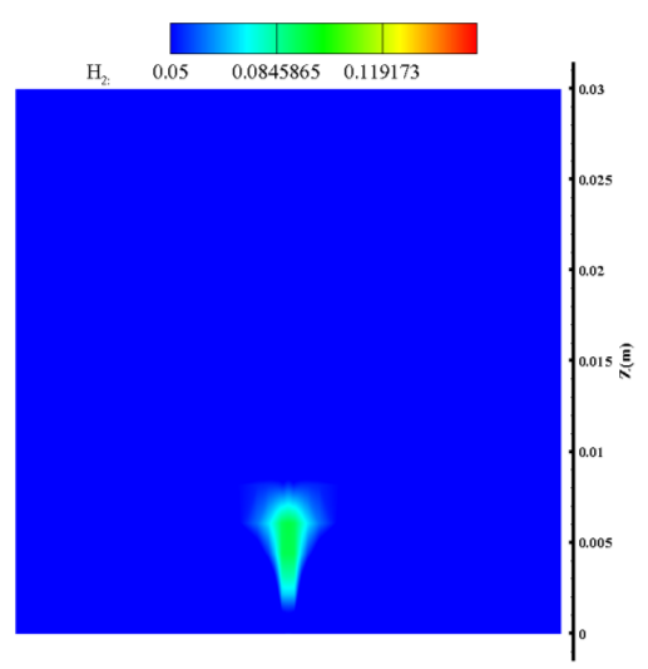

(d) $P_{\mathrm{b}} / P_{\infty}=8.0$

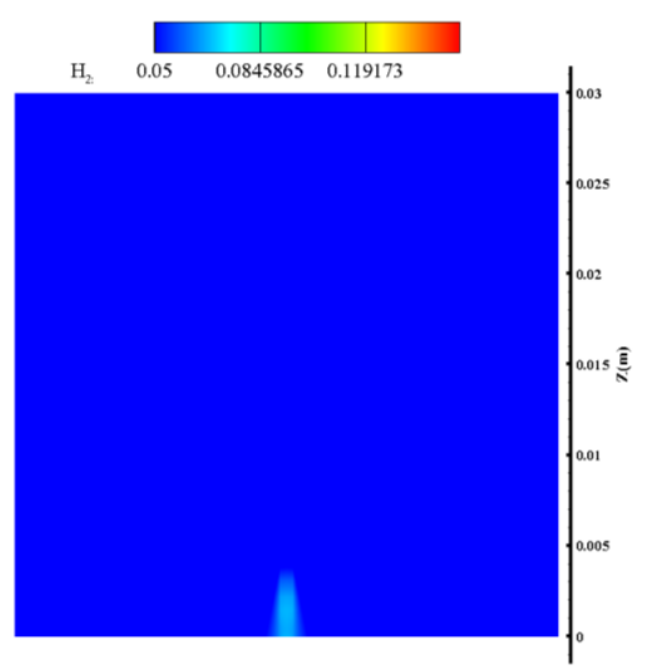

(f) $P_{\mathrm{b}} / P_{\infty}=15.0$

Fig. 8 Hydrogen mole fraction contour comparison at $x=20 \mathrm{~mm}$ with different back pressure ratios, (a) $P_{\mathrm{b}} / P_{\infty}=$ 


$$
\text { 3.0, (b) } P_{\mathrm{b}} / P_{\infty}=5.0 \text {, (c) } P_{\mathrm{b}} / P_{\infty}=8.0, \text { (d) } P_{\mathrm{b}} / P_{\infty}=10.0 \text { and (e) } P_{\mathrm{b}} / P_{\infty}=15.0 \text {. }
$$

The mixing efficiency is employed to evaluate the influence of the back pressure on the mixing enhancement in the transverse gaseous injection flow field, and its definition is as follows [42].

$$
\eta_{m}=\frac{\dot{m}_{\text {fuel, } \text { mixed }}}{\dot{m}_{\text {fuel,total }}}=\frac{\int \alpha_{\text {react }} \rho u d A}{\int \alpha \rho u d A}
$$

Herein,

$$
\alpha_{\text {react }}=\left\{\begin{array}{c}
\alpha, \alpha \leq \alpha_{\text {stoic }} \\
\alpha(1-\alpha) /\left(1-\alpha_{\text {stoic }}\right), \alpha>\alpha_{\text {stoic }}
\end{array}\right.
$$

$\alpha$ is injectant mass fraction, $\alpha_{\text {react }}$ is the injectant fraction mixed in a proportion that can react, $\alpha_{\text {stoic }}$ is the injectant stoichiometric mass fraction, $\dot{m}_{f u e l, m i x e d}$ is the mixed injectant mass flow and $\dot{m}_{f u e l, t o t a l}$ is the total injectant flow rate. $\rho$ and $u$ are the local density and velocity respectively, and $A$ is the cross section of the axial station where mixing is evaluated.

Fig.9 depicts the mixing efficiency comparison for cases with different back pressure ratios. It is obvious that the mixing efficiencies for the cases with the back pressure ratio being 8.0,10.0 and 15.0 are the same, and the injectant and air mix completely even at $x=10 \mathrm{~mm}$. This is consistent with the hydrogen mole fraction distribution at the symmetric plane, namely the phenomenon observed in Fig.7. At the same time, it is observed that the mixing process between the injectant and air is enhanced by the pseudo shock wave induced by the high back pressure, and this may imply that the intense combustion process downstream of the wall orifice is beneficial for the mixing between the injectant and air. This conclusion is consistent with that observed experimentally by Cantu et al. [43]. In other words, the mixing and combustion process between the injectant and air can be enhanced mutually. 


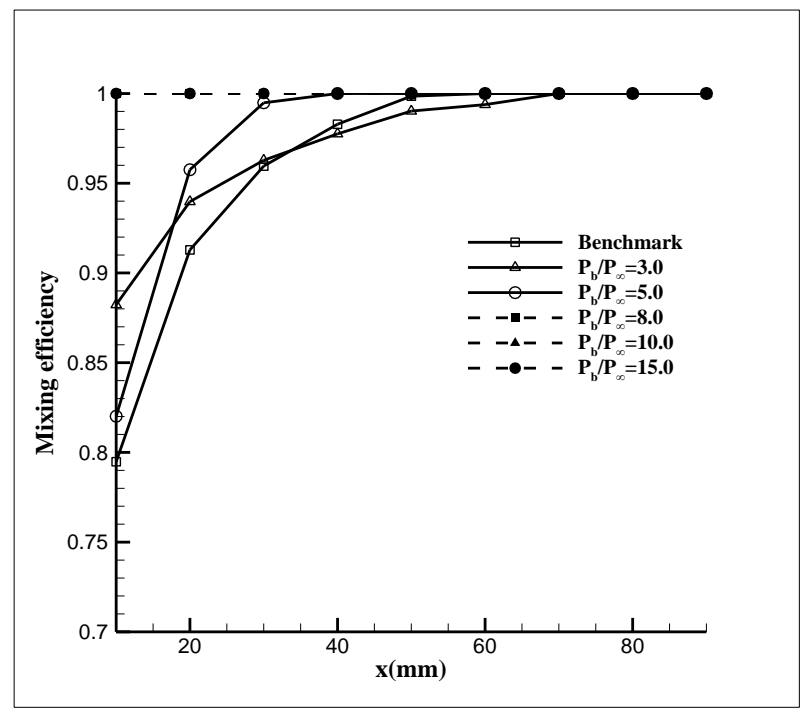

Fig.9 Mixing efficiency comparison for cases with different back pressure ratios.

\section{Conclusions}

In the current study, the transverse gaseous injection flow field with the pseudo shock wave has been investigated numerically, and the influence of the back pressure ratio on the flow field properties has been evaluated as well. The back pressure ratio is set to be as 3.0, 5.0, 8.0, 10.0 and 15.0. We have come to the following conclusions:

(1) The pseudo shock wave induced by the high back pressure has a large impact on the mixing enhancement between the injectant and air, and this implies that the intense combustion downstream of the wall orifice is beneficial to the mixing process. The mixing and combustion process between the injectant and air can be enhances mutually, and the mixing efficiency increases with the increase of the back pressure when the back pressure ratio is high enough, namely $P_{\mathrm{b}} / P_{\infty}=5.0$ in the range consider in this paper. When the back pressure ratio is 3.0, the near-field mixing process is improved when compared with the benchmark.

(2) Due to the fuel injection on one side of the flowpath, the flow field structure is not symmetric, and this is different from that observed in the scramjet isolator. When the pseudo shock wave occurs downstream of the injector, the hydrogen mole fraction distribution would be broken down by the shock wave train, and more fuel 
would penetrate into the core flow. However, when the pseudo shock wave has been pushed upstream of the injector, more injectant would be brought into the separation zone upstream of the wall orifice, and this is beneficial for the mixing process.

\section{Acknowledges}

The authors would like to express their thanks for the support from the National Science Foundation of China (No. 11502291) and a fund for owner of Outstanding Doctoral Dissertation from the Ministry of Education of China (No.201460). Also, the authors thank the anonymous reviewers for some very critical and constructive recommendations on this article.

\section{References}

1. Seiner J M, Dash S M, Kenzakowski D C. Historical survey on enhanced mixing in scramjet engines. Journal of Propulsion and Power, 2001, 17(6): 1273-1286

2. Glagolev A I, Zubkov A I, Panov Yu A. Supersonic flow past a gas jet obstacle emerging from a plate. Fluid Dynamics, 1967, 2(3): 60-64

3. Glagolev A I, Zubkov A I, Panov Yu A. Interaction between a supersonic flow and gas issuing from a hole in a plate. Fluid Dynamics, 1968, 3(2): 65-67

4. Huang W, Li S B, Yan L, Wang Z G. Performance evaluation and parametric analysis on cantilevered ramp injector in supersonic flows. Acta Astronautica, 2013, 84: 141-152

5. Huang W, Li S B, Yan L, Tan J G. Multiobjective design optimization of a cantilevered ramp injector using the surrogate-assisted evolutionary algorithm. Journal of Aerospace Engineering, 2015, 28: 04014138

6. Vergine F, Crisanti M, Maddalena L, Miller V, Gamba M. Supersonic combustion of pylon-injected 
hydrogen in high-enthalpy flow with imposed vortex dynamics. Journal of Propulsion and Power, 2015, 31(1): $89-103$

7. Vishwakarma M, Vaidyanathan A. Experimental study of mixing enhancement using pylon in supersonic flow. Acta Astronautica, 2016, 118: 21-32

8. Aguilera C, Yu K H. Supersonic mixing enhancement using fin-guided fuel injection. Journal of Propulsion and Power, 2015, 31(6): 1532-1543

9. Portz R, Segal C. Penetration of gaseous jets in supersonic flows. AIAA Journal, 2006, 44(10): 2426-2429

10. Kovar A, Schulein E. Comparison of experimental and numerical investigation on a jet in supersonic cross-flow. The Aeronautical Journal, 2006, 110: 353-360

11. Huang W, Liu W D, Li S B, Xia Z X, Liu J, Wang Z G. Influences of the turbulence model and the slot width on the transverse slot injection flow field in supersonic flows. Acta Astronautica, 2012, 73: 1-9

12. Huang W, Ma L, Pourkashanian M, Ingham D B, Luo S B, Wang Z G. Parametric effects in a scramjet engine on the interaction between the air stream and the injection. Proceedings of the Institution of Mechanical Engineers, Part G: Journal of Aerospace Engineering, 2012, 226: 294-309

13. Won S H, Jeung I S, Parent B, Choi J Y. Numerical investigation of transverse hydrogen jet into supersonic crossflow using detached-eddy simulation. AIAA Journal, 2010, 48(6): 1047-1058

14. Huang W, Yang J, Yan L. Multi-objective design optimization of the transverse gaseous jet in supersonic flows. Acta Astronautica, 2014, 93: 13-22

15. Chenault C F, Beran P S. K- $\varepsilon$ and Reynolds stress turbulence model comparisons for two-dimensional injection flows. AIAA Journal, 1998, 36(8): 1401-1412

16. Pudsey A S, Wheatley V, Boyce R R. Behavior of multiple-jet interactions in a hypersonic boundary layer. Journal of Propulsion and Power, 2015, 31(1): 144-155 
17. Huang W. Numerical investigation on staged sonic jet interaction mechanism in a supersonic cross flow. Proceedings of the Institution of Mechanical Engineers, Part G: Journal of Aerospace Engineering, 2014, 228(14): 2641-2651

18. Huang W, Yan L. Progress in research on mixing techniques for transverse injection flow fields in supersonic crossflows. Journal of Zhejiang University - Science A (Applied Physics \& Engineering), 2013, 14(8): 554-564

19. Kouchi T, Masuya G, Hirano K, Matsuo A, Tomioka S. Supersonic combustion using a stinger-shaped fuel injector. Journal of Propulsion and Power, 2013, 29(3): 639-647

20. Zhang Y, Wang B, Zhang H, Xue S. Mixing enhancement of compressible planar mixing layer impinged by oblique shock waves. Journal of Propulsion and Power, 2015, 31(1): 156-169

21. Schetz J A, Maddalena L, Burger S K. Molecular weight and shock-wave effects on transverse injection in supersonic flow. Journal of Propulsion and Power, 2010, 26(5): 1102-1113

22. Huang W, Wang Z G, Wu J P, Li S B. Numerical prediction on the interaction between the incident shock wave and the transverse slot injection in supersonic flows. Aerospace Science and Technology, 2013, 28: $91-99$

23. Huang W, Tan J G, Liu J, Yan L. Mixing augmentation induced by the interaction between the oblique shock wave and a sonic hydrogen jet in supersonic flows. Acta Astronautica, 2015, 117: 142-152

24. Gerdroodbary M B, Ganji D D, Amini Y. Numerical study of shock wave interaction on transverse jets through multiport injector arrays in supersonic crossflow. Acta Astronautica, 2015, 115: 422-433

25. Zare-Behtash H, Lo K H, Kontis K, Ukai T, Obayashi S. Transverse jet-cavity interactions with the influence of an impinging shock. International Journal of Heat and Fluid Flow, 2015, 53: 146-155

26. Huang W. Transverse jet in supersonic crossflows. Aerospace Science and Technology, 2016, 50: 183-195 
27. Huang W, Wang Z G, Pourkashanian M, Ma L, Ingham D B, Luo S B, Lei J, Liu J. Numerical investigation on the shock wave transition in a three-dimensional scramjet isolator. Acta Astronautica, 2011, 68: $1669-1675$

28. Smirnov N N, Nikitin V F, Shurekhdeli S A. Investigation of self-sustaining waves in metastable systems: deflagration-to-detonation transition. Journal of Propulsion and Power, 2009, 25(3): 593-608

29. Choi B, Takae K, Kouchi T, Masuya G. Turbulent characteristics for jet injected into supersonic flow with pseudo shock wave. Journal of Propulsion and Power, 2012, 28(5): 971-981

30. Boles J A, Edwards J R, Baurle R A. Large-eddy/Reynolds-averaged navier-stokes simulations of sonic injection into Mach 2 crossflow. AIAA Journal, 2010, 48(7): 1444-1456

31. Ogawa S, Choi B I, Masuya G, Takita K. Fuel mixing enhancement by precombustion shock wave. ISABE Paper 2001-1188, 2001

32. Kouchi T, Shibayama H, Masuya G. Assessments of jet penetrations into supersonic crossflow using high-speed framing schlieren images (in Japanese). Proceedings of the 40th Fluid Dynamics Conference/Aerospace Numerical Simulation Symposium 2008, Sendai, Japan, 2008

33. Aso S, Okuyama S, Kawai M, Ando Y. Experimental study on mixing phenomena in supersonic flows with slot injection. In: 29th Aerospace Sciences Meeting, Reno, Nevada, 1991, AIAA Paper 91-0016

34. Fluent Inc., Fluent 6.3 user's guide, Lebanon, NH, Fluent Inc, 2006

35. Erdem E, Kontis K. Numerical and experimental investigation of transverse injection flows. Shock Waves, 2010, 20: 103-118

36. Huang W. Design exploration of three-dimensional transverse jet in a supersonic crossflow based on data mining and multi-objective design optimization approaches. International Journal of Hydrogen Energy, 2014, 39: 3914-3925 
37. Huang W. Effect of jet-to-crossflow pressure ratio arrangement on turbulent mixing in a flowpath with square staged injectors. Fuel, 2015, 144: 164-170

38. Smirnov N N, Betelin V B, Shagaliev R M, et al. Hydrogen fuel rocket engines simulation using LOGOS code. International Journal of Hydrogen Energy, 2014, 39(20): 10748-10756

39. Smirnov N N, Betelin V B, Nikitin V F, et al. Accumulation of errors in numerical simulations of chemically reacting gas dynamics. Acta Astronautica, 2015, 117: 338-355

40. Ding F, Shen C B, Huang W, Liu J. Numerical validation and back-pressure effect on internal compression flows of typical supersonic inlet. The Aeronautical Journal, 2015, 119(1215): 631-645

41. Oberkampf W L. Verification and validation in computational fluid dynamics. Progress in Aerospace Sciences, 2002, 38: 209-272

42. Segal C. The scramjet engine processes and characteristics. Cambridge University Press, 2009

43. Cantu L M L, Gallo E C A, Cutler A D, Bathel B F, Danehy P M, Rockwell R D, Goyne C P, McDaniel J C. Visualization of simulated fuel-air mixing in a dual-mode scramjet. Journal of Propulsion and Power, in press, doi: 10.2514/1.B35760 